\title{
Directional Dark Matter Searches with CYGNO
}

\author{
Fernando Domingues Amaro ${ }^{1}$, Elisabetta Baracchini ${ }^{2,3, *}$, Luigi Benussi ${ }^{4}$, Stefano Bianco ${ }^{4}$, Cesidio Capoccia ${ }^{4}$, \\ Michele Caponero ${ }^{4,5}$, Gianluca Cavoto ${ }^{6,7}$, André Cortez ${ }^{2,3} \mathbb{D}^{\text {, Igor Abritta Costa }}{ }^{8}$, Emiliano Dané ${ }^{4}$, Giorgio \\ Dho $^{2,3}$, Emanuele Di Marco ${ }^{6}$, Giulia D’Imperio ${ }^{6}$, Flaminia Di Giambattista 2,3, Robert R. M. Gregorio ${ }^{9}$, \\ Francesco Iacoangeli ${ }^{6}$, Herman Pessoa Lima Júnior ${ }^{10}$, Amaro da Silva Lopes Júnior ${ }^{8}$, Giovanni Maccarrone ${ }^{4}$, \\ Rui Daniel Passos Mano ${ }^{1}$, Michela Marafini ${ }^{11}$, Giovanni Mazzitelli ${ }^{4}$, Alasdair G. McLean ${ }^{9}$, Andrea Messina ${ }^{6,7}$, \\ Cristina Maria Bernardes Monteiro ${ }^{1}$, Rafael Antunes Nobrega ${ }^{8}$, Igor Fonseca Pains ${ }^{8}$, Emiliano Paoletti ${ }^{4}$, \\ Luciano Passamonti ${ }^{4}$, Sandro Pelosi ${ }^{6}$, Fabrizio Petrucci ${ }^{12,13}$, Stefano Piacentini ${ }^{6,7}$, Davide Piccolo ${ }^{4}$, \\ Daniele Pierluigi ${ }^{4}$, Davide Pinci ${ }^{6} \mathbb{D}$, Atul Prajapati ${ }^{2,3}$, Francesco Renga ${ }^{6} \mathbb{D}$, Rita Joana da Cruz Roque ${ }^{1}$, \\ Filippo Rosatelli ${ }^{4}$, Andrea Russo ${ }^{4}$, Joaquim Marques Ferreira dos Santos ${ }^{1}$, Giovanna Saviano ${ }^{4,14}$, Neil Spooner ${ }^{9}$, \\ Roberto Tesauro ${ }^{4}$, Sandro Tomassini ${ }^{4}$ (D) and Samuele Torelli ${ }^{2,3}$
}

1 LIBPhys, Department of Physics, University of Coimbra, 3004-516 Coimbra, Portugal; famaro@uc.pt (F.D.A.); cristinam@uc.pt (C.M.B.M.); ritaroque@fis.uc.pt (R.J.d.C.R.); RDPMano@uc.pt (R.D.P.M.); jmf@uc.pt (J.M.F.d.S.)

2 Gran Sasso Science Institute, 67100 L'Aquila, Italy; andre.f.cortez@gmail.com (A.C.); giorgio.dho@gssi.it (G.D.); flaminia.digiambattista@gssi.it (F.D.G.); atul.prajapati@gssi.it (A.P.); samuele.torelli@gssi.it (S.T.)

3 Laboratori Nazionali del Gran Sasso, Istituto Nazionale di Fisica Nucleare, 67100 Assergi, Italy

4 Laboratori Nazionali di Frascati, Istituto Nazionale di Fisica Nucleare, 00044 Frascati, Italy;

check for updates

Citation: Amaro, F.D.; Baracchini, E.; Benussi, L.; Bianco, S.; Capoccia, C.; Caponero, M.; Cavoto, G.; Cortez, A.; Costa, I.A.; Dané, E.; et al. Directional Dark Matter Searches with CYGNO. Particles 2021, 4 , 343-353.

https://doi.org/10.3390/particles4030029

Academic Editors: Diego González-Díaz and Paul Colas

Received: 31 May 2021

Accepted: 3 July 2021

Published: 6 July 2021

Publisher's Note: MDPI stays neutral with regard to jurisdictional claims in published maps and institutional affiliations.

Copyright: (c) 2021 by the authors. Licensee MDPI, Basel, Switzerland. This article is an open access article distributed under the terms and conditions of the Creative Commons Attribution (CC BY) license (https:// creativecommons.org/licenses/by/ $4.0 /)$. luigi.benussi@lnf.infn.it (L.B.); stefano.bianco@lnf.infn.it (S.B.); Cesidio.Capoccia@lnf.infn.it (C.C.); caponero@frascati.enea.it (M.C.); Emiliano.Dane@lnf.infn.it (E.D.); giovanni.maccarrone@lnf.infn.it (G.M.); giovanni.mazzitelli@lnf.infn.it (G.M.); Emiliano.Paoletti@lnf.infn.it (E.P.); luciano.passamonti@lnf.infn.it (L.P.); Davide.Piccolo@lnf.infn.it (D.P.); Daniele.Pierluigi@lnf.infn.it (D.P.); filippo.rosatelli@lnf.infn.it (F.R.); arusso@lnf.infn.it (A.R.); giovanna.saviano@cern.ch (G.S.); Roberto.Tesauro@lnf.infn.it (R.T.); sandro.tomassini@lnf.infn.it (S.T.)

5 ENEA Centro Ricerche Frascati, 00044 Frascati, Italy

6 Istituto Nazionale di Fisica Nucleare, Sezione di Roma, 00185 Rome, Italy; gianluca.cavoto@roma1.infn.it (G.C.); emanuele.dimarco@roma1.infn.it (E.D.M.); giulia.dimperio@roma1.infn.it (G.D.); francesco.iacoangeli@roma1.infn.it (F.I.); andrea.messina@uniroma1.it (A.M.); Alessandro.Pelosi@roma1.infn.it (S.P.); stefano.piacentini@uniroma1.it (S.P.); davide.pinci@roma1.infn.it (D.P.); francesco.renga@roma1.infn.it (F.R.)

7 Dipartimento di Fisica, Università La Sapienza di Roma, 00185 Roma, Italy

8 Faculdade de Engenharia, Universidade Federal de Juiz de Fora, Juiz de Fora 36036-900, MG, Brazil; igorabritta@gmail.com (I.A.C.); rafael.nobrega@ufjf.edu.br (R.A.N.); igor.pains@ufjf.edu.br (I.F.P.); silva.lopes@ufjf.edu.br (A.d.S.L.J.)

9 Department of Physics and Astronomy, University of Sheffield, Sheffield S3 7RH, UK; robert.gregorio@sheffield.ac.uk (R.R.M.G.); ali.mclean@sheffield.ac.uk (A.G.M.); n.spooner@sheffield.ac.uk (N.S.)

10 Brazilian Center for Research in Physics, Rio de Janeiro 22290-180, RJ, Brazil; herman.lima.jr@gmail.com

11 Museo Storico della Fisica e Centro Studi e Ricerche "Enrico Fermi", Piazza del Viminale 1, 00184 Roma, Italy; Michela.Marafini@roma1.infn.it

12 Dipartimento di Matematica e Fisica, Università Roma TRE, 00146 Roma, Italy; fabrizio.petrucci@uniroma3.it

13 Istituto Nazionale di Fisica Nucleare, Sezione di Roma TRE, 00146 Roma, Italy

14 Dipartimento di Ingegneria Chimica, Materiali e Ambiente, Sapienza Università di Roma, 00185 Roma, Italy

* Correspondence: elisabetta.baracchini@gssi.it

Abstract: The CYGNO project aims at developing a high resolution Time Projection Chamber with optical readout for directional dark matter searches and solar neutrino spectroscopy. Peculiar CYGNO's features are the 3D tracking capability provided by the combination of photomultipliers and scientific CMOS camera signals, combined with a helium-fluorine-based gas mixture at atmospheric pressure amplified by gas electron multipliers structures. In this paper, the performances achieved with CYGNO prototypes and the prospects for the upcoming underground installation at Laboratori Nazionali del Gran Sasso of a 50-L detector in fall 2021 will be discussed, together with the plans for a 
1- $\mathrm{m}^{3}$ experiment. The synergy with the ERC consolidator, grant project INITIUM, aimed at realising negative ion drift operation within the CYGNO 3D optical approach, will be further illustrated.

Keywords: dark matter; time projection chamber; optical readout; electroluminescence; negative ion drift

\section{Introduction}

Dark Matter (DM) dominance of our universe is an established paradigm nowadays, and the identification and the study of its nature represent one of the most compelling tasks for fundamental physics. Weakly Interacting Massive Particles (WIMPs) are wellmotivated DM candidates, independently predicted by standard model extensions and Big Bang cosmology. Direct DM searches look for 1-100 keV Nuclear Recoils (NRs) induced by elastic scattering of WIMPs in the detector volume. The main experimental challenge is to positively discriminate low energy NRs from interactions induced by other particles, which have typically $10^{6}-10^{8}$ higher rates. The rarity of the expected interaction requires any backgrounds indistinguishable from the DM signal to be strictly controlled and eventually actively rejected in the data analysis. Classical background minimisation techniques are: Operation in deep underground laboratories (to suppress cosmic rays), use of radio-pure components (to avoid natural radioactivity), and active or passive shielding of the detector. Further background rejection can be achieved by exploiting the different material response to the energy deposition of nuclear or Electron Recoils (ERs).

The expected WIMP scattering in the detector is due to the Earth's relative motion with respect to the galactic halo that is believed to contain a high concentration of DM from the measurement of the rotational curves of our galaxy. This implies that a DM wind apparently coming from the Cygnus constellation is expected to be observable on our planet [1], with a change in direction of about 90 degrees for every 12 sidereal hours due to Earth's axis orientation with respect to the direction of motion of the solar system. The determination of the incoming direction of the DM particle can provide a correlation with an astrophysical source that no background whatsoever can mimic and therefore offers an unique key for a positive, unambiguous identification of a DM signal [2]. The capability of reconstructing recoils direction allows moreover to discriminate DM interactions from the scattering of solar neutrinos that would otherwise constitute an irreducible background, usually referred to as a "Neutrino Floor" [3]. Directional measurements can furthermore discriminate between various DM halo models and provide constraints on WIMP properties, like no other non-directional detector [2,4].

The CYGNO project proposes an innovative approach to direct DM searches, where a high resolution gaseous Time Projection Chamber (TPC) with light target nuclei and 3D tracking is employed, to boost sensitivity to $\mathrm{O}(\mathrm{GeV})$ masses, while at the same time maintaining directionality and background rejection down to low energy thresholds. The foreseen experiment capabilities to actively discriminate electromagnetic background and to determine both ERs and NRs direction of arrival will allow CYGNO to explore new physics cases that possess such signatures. These include, among the others, the elastic scattering of sub-GeV DM [5] and of solar neutrinos [6,7].

\section{Time Projection Chambers for Directional Dark Matter Searches}

TPCs can potentially provide the best observable and architecture for a DM search experiment. The total ionisation measured in a TPC indicates the energy of the recoil. Comparison between track path and energy provides excellent identification of alphas and electrons. The track itself indicates the axis of the recoil and a measurement of the charge (and $\mathrm{dE} / \mathrm{dx}$ ) released along its path allows for inferring the sense of direction, providing the required directionality. The topological signature of the recoil event improves also particle identification and hence background rejection. Thanks to recent advances in Micro 
Pattern Gas Detectors (MPGD) amplification and improved readout techniques, TPCs are moreover nowadays mature detectors to aim at developing a large scale experiment of $\mathrm{O}(100-1000) \mathrm{m}^{3}[8,9]$.

TPCs energy threshold and angular performances are eventually determined by the gas composition and pressure, and by the granularity and noise to the signal ratio of the amplification and readout system [4]. A comprehensive review of readout technologies for directional WIMP searches can be found in [10].

Direct DM search detectors lack the magnetic fields that are typically present in TPC installed within experiments at colliders or beams and that can be used to control diffusion over long drift distances. Given the need for large exposure, (hence detector volumes) a peculiar modification of the TPC operating principles has been developed within the DM field, called Negative Ion Drift (NID) [11,12] with the DRIFT experiment pioneering this innovation [13]. With NID, primary electrons liberated by the track ionising the gas are captured at $\mathrm{O}(\mu \mathrm{m})$ by the electronegative molecules, creating negative ions. The anions drift to the anode, where their additional electron is stripped and gives rise to a standard electron avalanche. Thanks to the anions mass being much larger than electrons, diffusion is reduced to the thermal limit with dispersion of $\mathrm{O}(1 \mathrm{~mm} / \mathrm{m})[11,12]$, significantly improving tracking.

Recently, a new remarkable feature has been observed in negative ion gas mixtures: The presence of multiple charge carriers in the time signal, with different masses [14]. Since anions mobility depends on their mass, the difference in time of arrival of different anions effectively provides a measurement of the position of the event along the drift direction. This is a feature that gaseous TPCs employed in DM searches can not usually grant, since they are not typically sensitive to the primary scintillation light nor possess other information on the time of the start of the event (in contrast to TPC at colliders/beams that can be triggered by the fast signal from other detectors). One of the most dangerous backgrounds for direct DM searches are Radon Progeny Recoils (RPRs) from U and Th traces in detector components, which can mimic WIMP interactions, especially when plating out at the cathode due to the geometry of the decay [15]. In order to reject these events the full 3D position of the track, including along the drift direction, needs to be reconstructed with a technique usually referred to as "fiducialization". Full 3D detector fiducialization can be obtained exploiting NID minority carriers time of arrival, and background-free operation over $1 \mathrm{~m}^{3}$ has been achieved by DRIFT thanks to this [13]. With these two features, NITPC readout planes can image a larger volume than conventional TPC approaches, resulting in lower backgrounds and costs for unit mass.

\section{The CYGNO TPC Optical 3D Approach}

The CYGNO innovative approach to TPC track imaging involves the optical detection of the photons produced by the de-excitation of gas molecules during the processes of electron multiplication [16]. The light yield and spectrum strongly depends on the gas and operating conditions $[17,18]$. Thanks to the recent developments in both the performance of Micro Pattern Gas Detectors and the evolution of the CMOS technology, this approach can today achieve very high granularity and tracking precision. CYGNO innovative technological and operational choices are:

- The use of Gas Electron Multipliers (GEMs), which provides very high and uniform gas gains combined with high granularity at the amplification stage;

- The use of scientific CMOS-based cameras (sCMOS) positioned behind the amplification plane for the detection of the scintillation light produced together with the electron avalanche in the GEMs. These devices offer a high granularity $2 \mathrm{D}$ readout along with very high sensitivity in the visible range ( $>90 \%$ of quantum efficiency at $600 \mathrm{~nm}$ ). sCMOS exhibit very low noise ( $\leq 1 \mathrm{e}^{-} /$pixel), have low power consumption, and offer larger field of view compared to Charged Coupled Devices $(\times 2.5$ than any standard EM-CCD) combined with smaller pixels $\left(6.5 \times 6.5 \mu \mathrm{m}^{2}\right)$ (for more details see https: //www.hamamatsu.com/eu/en/product/cameras/cmos-cameras/index.html, ac- 
cessed on 5 July 2021). Coupled to the proper optics, large areas can be imaged with a single sCMOS camera while maintaining a small effective pixels size (i.e., $160 \times$ $160 \mu \mathrm{m}^{2}$ for an area of $35 \times 35 \mathrm{~cm}^{2}$ ), favouring the scalability of this experimental approach. An example of images from Hamamatsu sCMOS cameras is shown in left panel of Figure 1, in particular a low energy electron in the top, and a nuclear recoil in the bottom;

- The combination of the sCMOS 2D X-Y track projection with the signal from a Photomultiplier (PMT) for the detection of the time profile of charge arrival. Thanks to this, the relative track extension along the drift direction $(\Delta Z)$ and the inclination with respect to the amplification plane can be inferred, and $3 \mathrm{D}$ reconstruction can be achieved with $\mathrm{O}(100) \mu \mathrm{m}$ precision [19]. An example of the PMT signal for tracks with different inclination with respect to the drift direction is shown in the right panel of Figure 1;

- The use of an He: $\mathrm{CF}_{4}$ 60:40 gas mixture at $1 \mathrm{~atm}$, with spectral emission at $600 \mathrm{~nm}$ nicely matching the sCMOS camera sensitivity. Given the kinematic of the WIMPnucleus elastic process, a direct DM detection experiment achieves its best sensitivity for WIMP masses equal to the target mass nuclei [20]. Thanks to its low density and atomic number, the use of helium can hence extend CYGNO sensitivity to low $\mathrm{O}(\mathrm{GeV})$ WIMP masses for spin independent coupling and allow to reach atmospheric pressure operation while maintaining good tracking. The $\mathrm{CF}_{4}$ is added for its well-known scintillation properties in the visible region $[17,18]$, where sCMOS cameras have the maximum sensitivity. In addition, $\mathrm{CF}_{4}$ large Fluorine content provide a spin-odd target for simultaneous sensitivity to spin dependent WIMP-proton interactions;

- The atmospheric pressure and room temperature operation; while increasing the available target mass compared to current gaseous directional DM approaches, it also minimises the infrastructures needed (no need for cryogenics as noble liquids or bolometer detectors) and overall experiment dimensions, costs, and material budget (thin vessel compared to all other low/high pressure TPC approaches);

- The decoupling of the readout sensor from the gas target volume, not possible with charge-based readouts. This avoids the readout outgassing into the target, modifying the gas properties or producing recoiling radon progeny in or near the active detector volume, relatively relaxing the radiopurity requirements;

- The possibility to improve tracking and fiducialization performances with the use of negative ion drift gas mixture; here lies the cardinal synergy between CYGNO and INITIUM. The challenging goal of the INITIUM project (funded as the ERC Consolidator, grant 2018, proposal no. 818744) is to develop NID operation at $1 \mathrm{~atm}$ within the CYGNO optical approach by the addition of a small quantity of $\mathrm{SF}_{6} . \mathrm{SF}_{6}$ has been recently demonstrated to work very well as negative ion gas between 20 and 100 Torr, including the possibility of high gains and fiducialization via minority charge carriers [21-23]. Compared to other gases typically employed to induce NID, $\mathrm{SF}_{6}$ has the substantial advantages of safer handling, combined with easier Radon purification and recirculation [24]. With charge pixels readout and triple-thin GEMs, the feasibility of NID has alredy been demonstrated with $\mathrm{He}: \mathrm{CF}_{4}: \mathrm{SF}_{6}$ at 360:240:10 Torr at nearly atmospheric pressure (0.8 atm) [25].

The recent developments in the MPGD amplification field combined with the fast advancement of CMOS technology, strongly pulled by the market, allows to foresee future developments for a large scale detector (CYGNO PHASE-2), with a staged approach passing through underground installation of a 50-L prototype (CYGNO PHASE-0) followed by a 1- $\mathrm{m}^{3}$ demonstrator (CYGNO PHASE-1). With this program, CYGNO fits in the context of the wider international CYGNUS effort, to establish a multi-modular, multi-target Galactic Directional Recoil Observatory based on gaseous TPCs, that can test the DM hypothesis beyond the Neutrino Floor and measure the coherent scattering of neutrinos from the sun and supernovae [4]. CYGNO is part of the coordinated R\&D effort to optimise technologies 
and gas mixture choices towards CYGNUS development that is currently undergoing in participating countries.
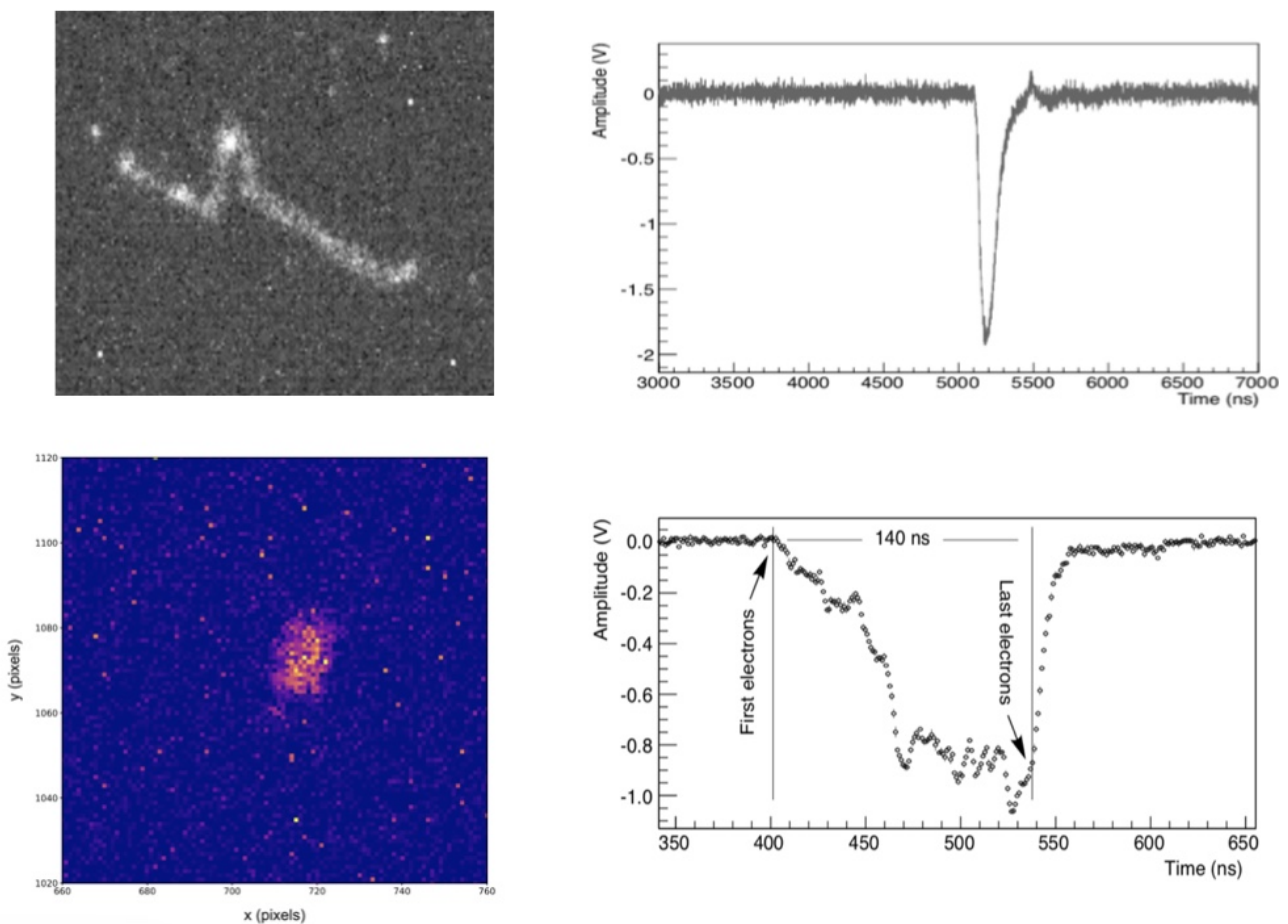

Figure 1. Examples of the CYGNO signals from the sCMOS (left) and the PMT (right) from tracks produced by particles interacting with an $\mathrm{He}: \mathrm{CF}_{4}$ 60:40 gas mixture at $1 \mathrm{~atm}$. In the left panel, images of a low energy electron from natural radioactivity on the top and a nuclear recoil on the bottom. In the right panel, PMT signals from tracks parallel (top) and tilted (bottom) with respect to the GEMs amplification plane.

\section{Experimental Results Obtained with CYGNO Prototypes}

Three prototypes of different dimensions have been developed within the CYGNO effort, to each focus on different sets of study.

The smaller detector called MANGO, schematically shown in left Figure 2, comprises of a $10 \times 10 \mathrm{~cm}^{2}$ GEMs amplification area and a 1-cm drift gap equipped with the Hamamatsu Orca Fusion sCMOS $\left(2304 \times 2304\right.$ pixels of $\left.6.5 \times 6.5 \mu \mathrm{m}^{2}\right)$ and one PMT, and is employed to study innovative gas mixtures and amplification strategies for improved light yield and reduced diffusion. The sCMOS, equipped with a Schneider lens ( $\mathrm{f}=0.95$ and $25 \mathrm{~mm}$ focal length), is placed at a distance of $27.5 \mathrm{~cm}$ from the last GEM in order to image an area of $13.3 \times 13.3 \mathrm{~cm}^{2}$, with $65 \times 65 \mu^{2}$ effective pixel size. With MANGO, the first experimental evidence of luminescence in $\mathrm{He}: \mathrm{CF} 4$ gas mixtures induced by non-ionising electrons was obtained [26]. To this aim, a metal mesh was placed $2.5 \mathrm{~mm}$ beyond the last GEM (shown in Figure 2, left inset). This is used to create an electric field to accelerate the avalanche electrons to induce additional light production from gas molecules. With this and an applied electric field of $11.3 \mathrm{kV} / \mathrm{cm}$ in the gap, a factor 3 increase of the recorded light was observed, without any significant increase of the charge produced nor deterioration of the energy resolution. This feature can provide significant increases of the scintillation yield without the need for large gas amplification gain, helping in reducing the experimental energy threshold while improving the energy resolution. While this phenomenon is well known and already exploited in Argon- and Xenon-based detectors; this is the first time it has been observed with Helium and $\mathrm{CF}_{4}$. 

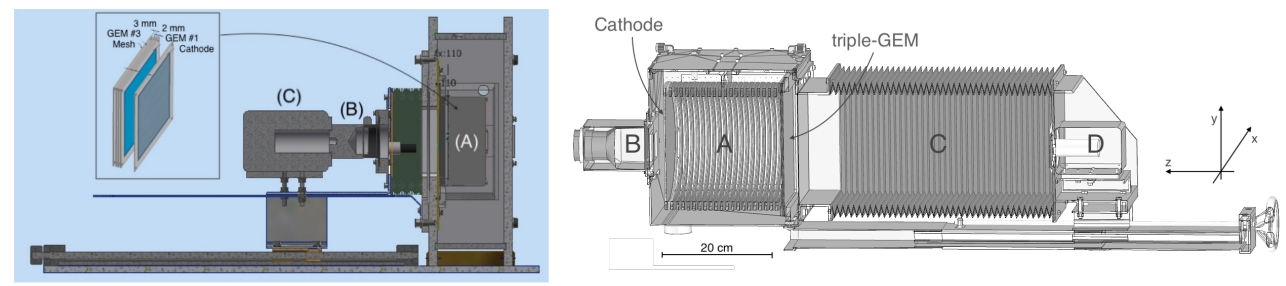

Figure 2. Schematic of CYGNO prototypes experimental setup. In the left panel, MANGO with sensitive volume (A), lens (B), and the CMOS camera (C); in the right panel, LEMOn with elliptical sensitive volume (A), PMT (B), optical bellow (C), and the sCMOS camera (D).

The medium-size prototype LEMOn, schematically shown on the right in Figure 2, is composed by a 7-L gas sensitive volume (A), enclosed in an elliptical field cage with a 20-cm drift length and, a $24 \times 20 \mathrm{~cm}^{2}$ base, to match the rectangular triple GEMs amplification stage dimensions. A mesh-based semitransparent cathode closes the volume on the opposite side, and the whole structure is contained in a gas-tight box with two transparent windows on the GEMs and cathode sides, behind which a PMT is placed (B). Behind the last GEM, downstream to an adjustable bellow (C), a Hamamatsu ORCA Flash $4.0 \mathrm{sCMOS}$ camera $\left(2048 \times 2048\right.$ pixels of $\left.6.5 \times 6.5 \mu \mathrm{m}^{2}\right)$ is placed at distance of $52.5 \mathrm{~cm}$. With the Schneider lens, it proves a Field of View (FOV) of $26 \times 26 \mathrm{~cm}^{2}$ with $130 \times 130 \mu \mathrm{m}^{2}$ effective pixel size. A more detailed description of the LEMOn prototype can be found in Ref. $[27,28]$.

With LEMOn, CYGNO experimental approaches performances were assessed on a medium-size scale in overground Laboratori Nazionali di Frascati (LNF) with the use of radioactive sources and cosmic rays. In particular:

- Detector stability was evaluated operating continuously the detector for a month-long test [29]. The currents drawn by the high voltage channels supplying the electrodes of the GEM stack were monitored and recorded to identify sudden and large increases that could indicate discharges or other electrostatic issues. The autorecovery procedure implemented resulted in a dead time of less than $4 \%$. Overall, the test demonstrated the high stability of the experimental approach;

- Light yield, energy resolution, and detection efficiency at $\mathrm{O}(\mathrm{keV})$ was studied analysing the sCMOS images of ${ }^{55} \mathrm{Fe} X$-rays-induced electron recoils. A response of about 514 photons/ $\mathrm{keV}$ was measured with LEMOn, with $12 \%$ energy resolution $[27,29]$ from the ${ }^{55} \mathrm{Fe}$ spectrum show on the left of Figure 3 and full detection efficiency in the whole 7-L volume;

- Detection threshold was evaluated studying the sCMOS sensor noise in absence of light. Requiring that less than 10 fake events/year are reconstructed from sensor noise translate in a detection threshold of $1 \mathrm{keV}$ [27];

- Event absolute drift distance estimation from the fit to diffusion, as demonstrated with charge pixels readout by [30], was studied with $450 \mathrm{MeV}$ electrons from the LNF-BTF facility [28], for which the start time of the event can be known from the beam trigger. The transverse recorded light profile by both the sCMOS camera and PMT shows a dependence as a function of the absolute drift distance that can be used to infer the absolute $\mathrm{Z}$ with $15 \%$ uncertainty over a 20 -cm length [28];

- Detection and identification of nuclear and electron recoils was assessed with a dedicated iterative algorithm (iDBSCAN) [31] developed from well-known Density-Based Spatial Clustering of Applications with Noise (DBSCAN) [32] aimed at recognising tracks with different ionisation patterns. With iDBSCAN applied on electron recoils from ${ }^{55} \mathrm{Fe}$ and nuclear recoils induced by $\mathrm{AmBe}$, a $10^{-3} \mathrm{ER}$ rejection at $5.9 \mathrm{keV}_{e e}$ with $40 \%$ NR efficiency was obtained with an elementary cut [33], a result that can be significantly improved by the use of a multivariate approach. 

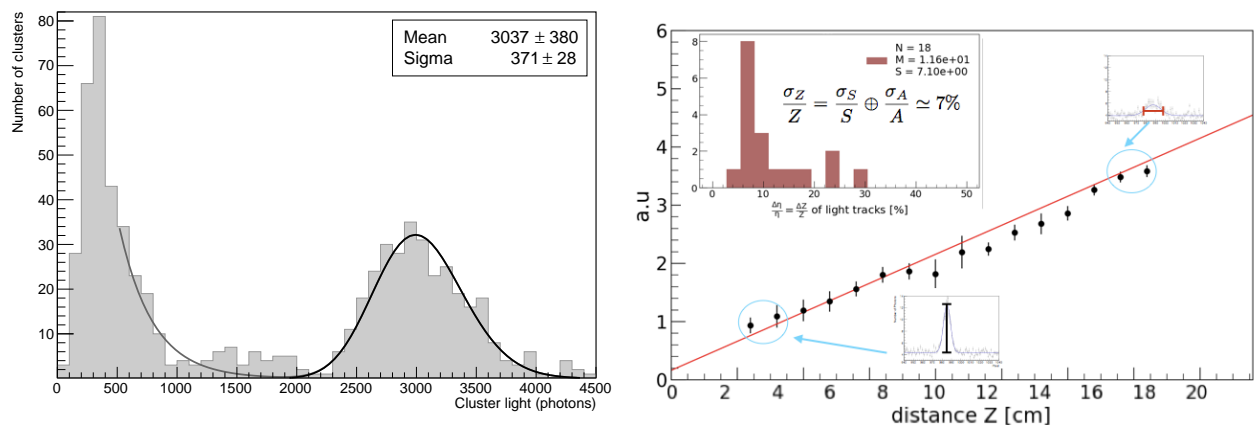

Figure 3. Left panel: Light spectrum of ${ }^{55} \mathrm{Fe}$ events reconstructed from the sCMOS images with Polya fit superimposed from [27]. Right panel: $\eta_{\text {light }}$ (see text for details) as a function of the drift distance $\mathrm{Z}$ measured with $450 \mathrm{MeV}$ electrons from the LNF-BTF facility [28].

LIME, shown in Figure 4, is the largest detector manufactured so far by the collaboration and represents the CYGNO PHASE-0 mentioned in Section 3. LIME is equipped with triple $33 \times 33 \mathrm{~cm}^{2}$ thin GEMs, amplifying a 50-cm drift length, for a total active volume of about $55 \mathrm{~L}$ imaged by a single sCMOS and a 4 small PMT. The GEMs are stretched with pullouts, following the experience gained within the CMS experiment [34], on a plexiglass frame rather than on the conventional CERN PCBs (bottom right of Figure 4), in order to minimise the radioactivity contribution from this item. The field cage is composed by copper rings supported by a plexiglass comb (Figure 4, bottom left), conveniently roundly shaped to avoid discharge at a $1-\mathrm{cm}$ pitch. A plexiglass box contains the detector $55-\mathrm{L}$ active volume guaranteeing gas tightness, with a thin Mylar window for calibrations with radioactive sources and a laser beam. An aluminum Faraday cage envelope the detector to protect from electric noise and discharges (Figure 4 top right). LIME possess the same dimensions of a single module of the 18 foreseen for a $1-\mathrm{m}^{3}$ CYGNO PHASE-1 detector (see Section 5). The LIME goal is in fact to verify in an underground environment and on realistic dimensions, the performances expected for CYGNO PHASE-1, while at the same time test part of the materials and construction techniques that will be employed for the realisation of the experiment.

LIME has been commissioned in overground LNF and demonstrated a detector stability consistent with the one measured on LEMOn [29]. LIME is read out with the Hamamatsu ORCA Fusion sCMOS, that improves over the LEMOn Orca Flash in terms of reduced noise, larger number of pixels, and larger quantum efficiency. The Orca Fusion has a FOV of $35 \times 35 \mathrm{~cm}^{2}$, providing effective pixels the size of $160 \times 160 \mu \mathrm{m}^{2}$. With this configuration, a response of $1.18 \mathrm{ph} / \mathrm{eV}$ and $<10^{-5}$ fake clusters per year was measured in LIME, showing that detection energy threshold could be lowered from $1 \mathrm{keV}$ to $0.5 \mathrm{keV}$ with respect to the published measurement [27]. The energy resolution on the ${ }^{55} \mathrm{Fe}$ peak is measured to be $14 \%$ across the whole $50-\mathrm{cm}$ drift length, with $100 \%$ efficiency in the full 50-L volume. Measurements are ongoing to assess absolute $\mathrm{Z}$ resolution and ER/NR discrimination within LIME prototype. LIME location in the service tunnel connecting the LNGS Halls is under space optimisation and its underground installation is foreseen for fall 2021.

A summary of the main characteristics and achieved performances of the CYGNO large prototypes LEMOn and LIME discussed in the text are shown in Table 1.

Table 1. Summary of the main characteristics and achieved performances of the CYGNO large prototypes LEMOn and LIME discussed in the text.

\begin{tabular}{cccccccc}
\hline & FOV $\left[\mathrm{cm}^{2}\right]$ & Length $[\mathrm{cm}]$ & Light Yield & Det. Thr. & Energy Res. & Abs. Z Res. & ER/NR Discr. \\
\hline LEMOn & $26 \times 26$ & 20 & $514 \gamma / \mathrm{keV}_{e e}$ & $1 \mathrm{keV}_{e e}$ & $12 \% @ 5.9 \mathrm{keV}_{e e}$ & $15 \%$ & $10^{-3} @ 5.9 \mathrm{keV}_{e e}$ \\
LIME & $35 \times 35$ & 50 & $1180 \gamma / \mathrm{keV}_{e e}$ & $0.5 \mathrm{keV}_{e e}$ & $14 \% @ 5.9 \mathrm{keV}_{e e}$ & & \\
\hline
\end{tabular}




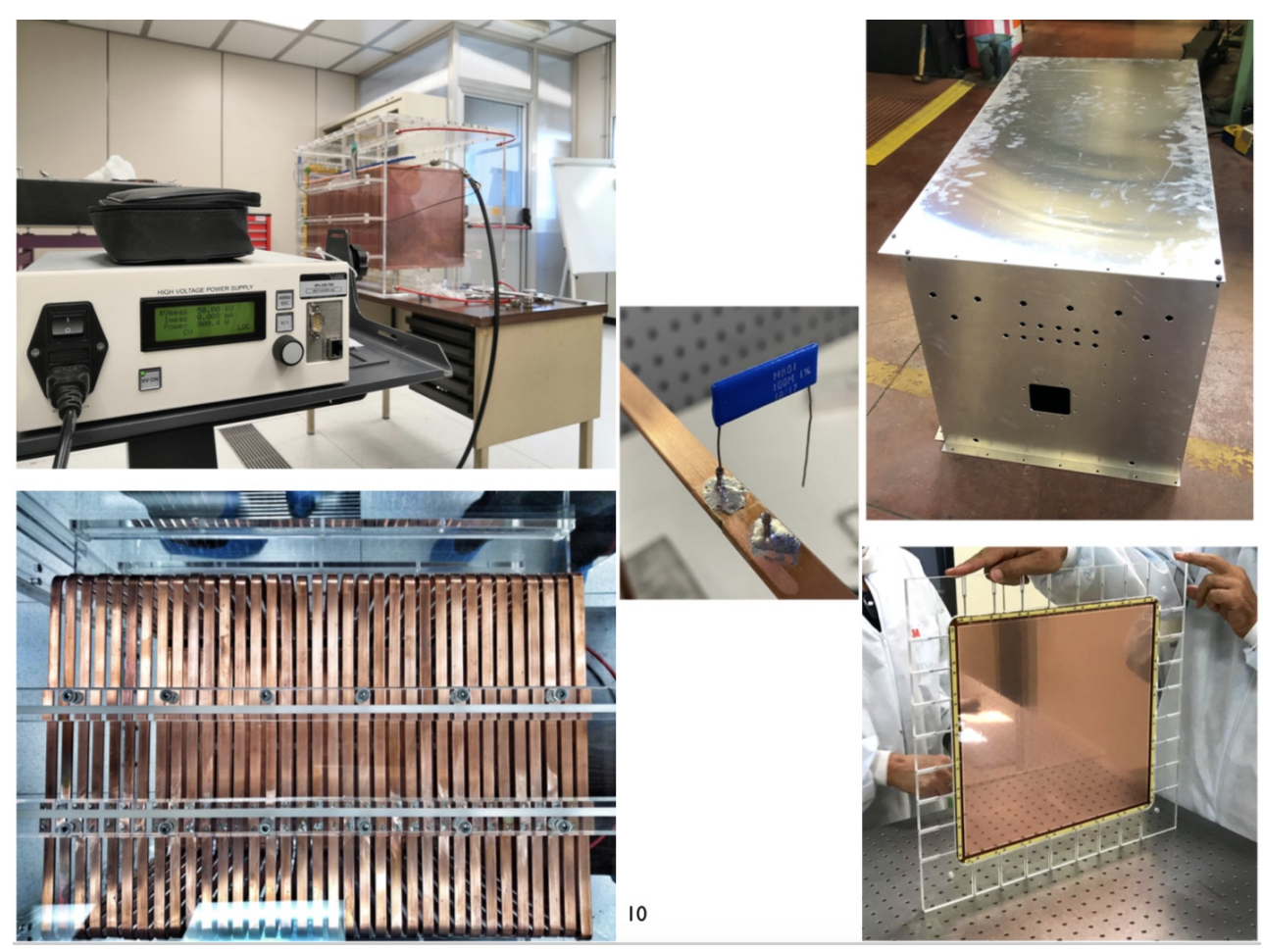

Figure 4. Picture of the LIME detector assembly at Laboratori Nazionali di Frascati. Top left: With drift field and GEM operating at nominal voltages, bottom left: Detail of the field cage copper rings, center: Detail of the resistor soldered to the field cage ring, top right: LIME inside the Faraday cage, and bottom right: Detail of the triple GEM streatched with pullouts on a plexiglass frame.

\section{The CYGNO PHASE-1 Detector}

CYGNO PHASE-1 detector will be composed of two back-to-back TPCs, separated by a central aluminised mylar cathode [35], for an active gas volume of the order of $1 \mathrm{~m}^{3}$. Each end cap will be equipped with $N$ LIME-like modules, where the actual number $N$ will depend on the final detector dimensions, still under optimisation. The field cage will be realised along the model developed for LIME, with a $6 \mathrm{~mm} \times 2 \mathrm{~mm}$ copper rings placed every $10 \mathrm{~mm}$. A gas volume vessel realised in PMMA to lower the material intrinsic radioactivity, gas contamination, and ensure the electrical isolation from cathode and filed cage, will contain the active volume of the detector. CYGNO PHASE-1 will be equipped with a dedicated DAQ system based on FPGA and CameraLink protocols to simultaneously acquire both the fast PMTs signals and slow CMOS cameras pictures only when their output is consistent with the presence of a track, and to synchronously store them for offline processing. CYGNO PHASE-1 will feature a tailored gas system, designed to performs four functions: Flow of a suitable gas mixture $\left(\mathrm{He}: \mathrm{CF}_{4}\right.$, typically in the ratio 60:40), chemical purification of impurities, recirculation, and recovery. The gas will be completely recovered from the system in order to produce no exhaust into the atmosphere. Both the DAQ and the gas systems are under finalisation and will be tested in the upcoming underground LIME installation (see Section 4).

Preliminary evaluation of the expected CYGNO PHASE-1 backgrounds has been performed with a Geant-4 [36] based Monte Carlo simulation. An external passive shielding of 2-m Water $+5 \mathrm{~cm}$ copper has been identified as the optimal configuration to reduce the number of expected ERs from LNGS environmental gamma and neutron fluxes below $10^{3}$ cpy (with $\mathrm{O}(1)$ cpy nuclear recoils) in the range $0-20 \mathrm{keV}$ [37]. The GEMs foil, the gas vessel PMMA, and the camera body and lens, expected to introduce the largest radioactivity contamination from internal components, have been measured with HPGe gamma spectroscopy detectors. Their activities were introduced in the PHASE-1 background simulation, obtaining a preliminary estimate of $\mathrm{O}\left(10^{6}\right)$ /year ERs induced by material radioactivity in the $0-20 \mathrm{keV}$ energy range before applying any discrimination. Background 
minimisation with the use of Suprasil lens $\left(\sim 10^{4}\right.$ reduction) and the substitution of part of the internal sCMOS camera components with cleaner options is under investigation in collaboration with the producing companies.

\section{Conclusions}

This paper illustrates the developments and plans for the realisation of CYGNO, a directional DM experiment based on optical readout of a TPC detector through the combination of sCMOS images and PMTs signals. Thanks to the recent developments in MPGD and CMOS technologies, gaseous TPCs, constituting the most natural approach to directional DM searches, have nowadays reached technological maturity to aim at large-scale experiments, as illustrated in [4]. CYGNO development fits into the CYGNUS proto-collaboration effort, with the goal of demonstrating the proof-of-principle of the chosen optical approach towards the realisation of a multi-modular ton-scale detector. The performances achieved with CYGNO prototypes and illustrated in the Section 4 results are highly promising. The realisation of a PHASE-1 detector based on this approach at underground Laboratori Nazionali del Gran Sasso is expected to significantly contribute to the advancement of TPC technology in the directional DM search field.

The development and optimisation of large high precision gaseous TPC with sCMOS + PMT 3D optical readout is of very high interest for several applications of TPC technology for rare event signatures other than DM searches [38]. These span from X-ray polarimetry in space [39], to low energy nuclear physics [40], to measurement of the angular correlations in the 2-proton decay [41], further demonstrating the versatility of the TPC approach, in particular when coupled to an innovative, highly-performing optical readout.

Author Contributions: Conceptualization: E.B., L.B., C.C., G.C., E.D., G.D. (Giorgio Dho), A.d.S.L.J., M.M., G.M. (Giovanni Mazzitelli), A.M., E.P., L.P., S.P., F.P., S.P., D.P. (Davide Piccolo), D.P. (Daniele Pierluigi), D.P. (Davide Pinci), F.R. (Francesco Renga), F.R. (Filippo Rosatelli), A.R., J.M.F.d.S., G.S., N.S., R.T. and S.T. (Sandro Tomassini); Data curation, A.C., G.D. (Giorgio Dho), E.D.M., I.F.P., D.P. (Davide Pinci), F.R. (Francesco Renga) and S.T. (Samuele Torelli); Formal analysis, F.D.A., C.M.B.M., R.J.d.C.R., I.A.C., G.D. (Giorgio Dho), E.D.M., F.D.G., F.I., H.P.L.J., A.G.M., I.F.P., D.P. (Davide Pinci), A.P. and S.T. (Samuele Torelli); Funding acquisition, E.B. and D.P. (Davide Pinci); Investigation, R.R.M.G., F.I., A.G.M., R.A.N., L.P. and D.P. (Davide Pinci); Methodology, F.D.A., E.B., L.B., C.M.B.M., C.C., M.C., G.C., A.C., G.D. (Giorgio Dho), E.D.M., G.D. (Giulia D’Imperio), F.D.G., R.R.M.G., H.P.L.J., G.M. (Giovanni Maccarrone), R.D.P.M., G.M. (Giovanni Mazzitelli), A.M., R.A.N., E.P., L.P., D.P. (Davide Piccolo), D.P. (Daniele Pierluigi), D.P. (Davide Pinci), F.R. (Francesco Renga), F.R. (Filippo Rosatelli), A.R., J.M.F.d.S., N.S., R.T. and S.T. (Sandro Tomassini); Project administration, E.B., G.C., G.M. (Giovanni Mazzitelli), D.P. (Davide Pinci) and F.R. (Francesco Renga); Resources, E.B. and D.P. (Davide Pinci); Software, G.D. (Giulia D'Imperio), F.D.G., F.P., S.P., A.P. and S.T. (Samuele Torelli); Supervision, E.B., L.B., S.B., C.C., G.M. (Giovanni Mazzitelli) and N.S.; Validation, F.D.A.; Visualization, E.B.; Writing—original draft, E.B.; Writing—review \& editing, S.B. and G.M. (Giovanni Maccarrone). All authors have read and agreed to the published version of the manuscript.

Funding: This project has received funding from the European Research Council (ERC) under the European Union's Horizon 2020 programme (grant agreement no. 818744).

Institutional Review Board Statement: Not applicable.

Informed Consent Statement: Not applicable.

Data Availability Statement: Not applicable.

Conflicts of Interest: The authors declare no conflict of interest.

\section{References}

1. Ahlen, S.; Afshordi, N.; Battat, J.B.R.; Billard, J.; Bozorgnia, N.; Burgos, S.; Caldwell, T.; Carmona, J.M.; Cebrian, S.; Colas, P.; et al. The case for a directional dark matter detector and the status of current experimental efforts. Int. J. Mod. Phys. A 2010, 25, 1-51. [CrossRef]

2. Mayet, F.; Billard, J. A review of the discovery reach of directional Dark Matter detection. Phys. Rept. 2016, 627, 1-49. [CrossRef] 
3. Billard, J.; Strigari, L.; Figueroa-Feliciano, E. Implication of neutrino backgrounds on the reach of next generation dark matter direct detection experiments. Phys. Rev. D 2014, 89, 023524. [CrossRef]

4. Vahsen, S.E.; O’Hare, C.A.J.; Lynch, W.A.; Spooner, N.J.C.; Baracchini, E.; Barbeau, P.; Battat, J.B.R.; Crow, B.; Deaconu, C.; Eldridge, C.; et al. CYGNUS: Feasibility of a nuclear recoil observatory with directional sensitivity to dark matter and neutrinos. arXiv 2020, arXiv:2008.12587.

5. Baracchini, E.; Derocco, W.; Dho, G. Discovering supernova-produced dark matter with directional detectors. Phys. Rev. D 2020, 102, 075036. [CrossRef]

6. Seguinot, J.; Ypsilantis, T.; Zichichi, A. A High rate solar neutrino detector with energy determination. Conf. Proc. C 1992, 920310, 289-313.

7. Arpesella, C.; Broggini, C.; Cattadori, C. A possible gas for solar neutrino spectroscopy. Astropart. Phys. 1996, 4, 333-341. [CrossRef]

8. Alme, J.; Andres, Y.; Appelshauser, H.; Bablok, S.; Bialas, N.; Bolgen, R.; Bonnes, U.; Bramm, R.; Braun-Munzinger, P.; Campagnolo, R.; et al. The ALICE TPC, a large 3-dimensional tracking device with fast readout for ultra-high multiplicity events. Nucl. Instrum. Meth. A 2010, 622, 316-367. [CrossRef]

9. Acciarri, R.; Acero, M.A.; Adamowski, M.; Adams, C.; Adamson, P.; Adhikari, S.; Ahmad, Z.; Albright, C.H.; Alion, T.; Amador, E.; et al. Long-Baseline Neutrino Facility (LBNF) and Deep Underground Neutrino Experiment (DUNE): Conceptual Design Report, Volume 4 The DUNE Detectors at LBNF. arXiv 2016, arXiv:1601.02984.

10. Battat, J.B.R.; Irastorza, I.G.; Aleksandrov, A.; Guler, M.A.; Asada, T.; Baracchini, E.; Billard, J.; Bosson, G.; Bourrion, O.; Bouvier, J.; et al. Readout technologies for directional WIMP Dark Matter detection. Phys. Rept. 2016, 662, 1-46. [CrossRef]

11. Martoff, C.J.; Snowden-Ifft, D.P.; Ohnuki, T.; Spooner, N.; Lehner, M. Suppressing drift chamber diffusion without magnetic field. Nucl. Instrum. Meth. A 2000, 440, 355-359. [CrossRef]

12. Ohnuki, T.; Snowden-Ifft, D.P.; Martoff, C.J. Measurement of carbon disulfide anion diffusion in a TPC. Nucl. Instrum. Meth. A 2001, 463, 142-148. [CrossRef]

13. Battat, J.B.R.; Ezeribe, A.C.; Gauvreau, J.-L.; Harton, J.L.; Lafler, R.; Law, E.; Lee, E.R.; Loomba, D.; Lumnah, A.; Miller, E.H.; et al. Low Threshold Results and Limits from the DRIFT Directional Dark Matter Detector. Astropart. Phys. 2017, 91, 65-74. [CrossRef]

14. Snowden-Ifft, D.P. Discovery of multiple, ionization-created CS2 anions and a new mode of operation for drift chambers. Rev. Sci. Instrum. 2014, 85, 013303. [CrossRef] [PubMed]

15. Pipe, M. Background reduction and spin-dependent limits using DRIFT: A directionally sensitive dark matter detector. J. Phys. Conf. Ser. 2011, 315, 012017. [CrossRef]

16. Dominik, W.; Zaganidis, N.; Astier, P.; Charpak, G.; Santiard, J.C.; Sauli, F.; Tribollet, E.; Geissbuhler, A.; Townsend, D. A Gaseous Detector for High Accuracy Autoradiography of Radioactive Compounds with Optical Readout of Avalanche Positions. Nucl. Instrum. Meth. A 1989, 278, 779. [CrossRef]

17. Fraga, M.M.F.R.; Fraga, F.A.F.; Fetal, S.T.G.; Margato, L.M.S.; Ferreira-Marques, R.; Policarpo, A.J.P.L. The GEM scintillation in He $\mathrm{CF}_{4}, \mathrm{Ar} \mathrm{CF}_{4}, \mathrm{Ar} \mathrm{TEA}$ and Xe TEA mixtures. Nucl. Instrum. Meth. 2003, A504, 88-92. [CrossRef]

18. Margato, L.M.S.; Morozov, A.; Fraga, M.M.F.R.; Pereira, L.; Fraga, F.A.F. Effective decay time of $\mathrm{CF}_{4}$ secondary scintillation. JINST 2013, 8, P07008. [CrossRef]

19. Antochi, V.C.; Baracchini, E.; Cavoto, G.; Marco, E.D.; Marafini, M.; Mazzitelli, G.; Pinci, D.; Renga, F.; Tomassini, S.; Voena, C. Combined readout of a triple-GEM detector. JINST 2018, 13, P05001. [CrossRef]

20. Gondolo, P. Recoil momentum spectrum in directional dark matter detectors. Phys. Rev. D 2002, 66, 103513. [CrossRef]

21. Phan, N.S.; Lafler, R.; Lauer, R.J.; Lee, E.R.; Loomba, D.; Matthews, J.A.J.; Miller, E.H. The novel properties of SF 6 for directional dark matter experiments. JINST 2017, 12, P02012. [CrossRef]

22. Ikeda, T.; Shimada, T.; Ishiura, H.; Nakamura, K.D.; Nakamura, T.; Miuchi, K. Development of a negative ion micro TPC detector with $\mathrm{SF}_{6}$ gas for the directional dark matter search. JINST 2020, 15, P07015. [CrossRef]

23. Lightfoot, P.K.; Spooner, N.J.C.; Lawson, T.B.; Aune, S.; Giomataris, I. First operation of bulk micromegas in low pressure negative ion drift gas mixtures for dark matter searches. Astropart. Phys. 2007, 27, 490-499. [CrossRef]

24. Ezeribe, A.C.; Lynch, W.; Gregorio, R.R.M.; Mckeand, J.; Scarff, A.; Spooner, N.J.C. Demonstration of radon removal from SF 6 using molecular sieves. JINST 2017, 12, P09025. [CrossRef]

25. Baracchini, E.; Cavoto, G.; Mazzitelli, G.; Murtas, F.; Renga, F.; Tomassini, S. Negative Ion Time Projection Chamber operation with $\mathrm{SF}_{6}$ at nearly atmospheric pressure. JINST 2018, 13, P04022. [CrossRef]

26. Baracchini, E.; Benussi, L.; Bianco, S.; Capoccia, C.; Caponero, M.; Cavoto, G.; Cortez, A.; Costa, I.A.; Marco, E.D.; D’Imperio, G.; et al. First evidence of luminescence in a $\mathrm{He} / \mathrm{CF}_{4}$ gas mixture induced by non-ionizing electrons. JINST 2020, 15 , P08018. [CrossRef]

27. Costa, I.A.; Baracchini, E.; Bellini, F.; Benussi, L.; Bianco, S.; Caponero, M.; Cavoto, G.; D’Imperio, G.; Marco, E.D.; Maccarrone, G.; et al. Performance of optically readout GEM-based TPC with a 55Fe source. J. Instrum. 2019, 14, P07011. [CrossRef]

28. Antochi, V.C.; Cavoto, G.; Costa, I.A.; Marco, E.D.; D’Imperio, G.; Iacoangeli, F.; Marafini, M.; Messina, A.; Pinci, D.; Renga, F.; et al. A GEM-based Optically Readout Time Projection Chamber for charged particle tracking. arXiv 2020, arXiv:2005.12272.

29. Costa, I.A.; Baracchini, E.; Bellini, F.; Benussi, L.; Bianco, S.; Caponero, M.; Cavoto, G.; D’Imperio, G.; Marco, E.D.; Maccarrone, G.; et al. Stability and detection performance of a GEM-based Optical Readout TPC with $\mathrm{He} / \mathrm{CF}_{4}$ gas mixtures. J. Instrum. 2020, 15, P10001. 
30. Lewis, P.M.; Vahsen, S.E.; Seong, I.S.; Hedges, M.T.; Jaegle, I.; Thorpe, T.N. Absolute Position Measurement in a Gas Time Projection Chamber via Transverse Diffusion of Drift Charge. Nucl. Instrum. Meth. A 2015, 789, 81-85. [CrossRef]

31. Baracchini, E.; Benussi, L.; Bianco, S.; Capoccia, C.; Caponero, M.; Cavoto, G.; Cortez, A.; Costa, I.A.; Marco, E.D.; D’Imperio, G.; et al. A density-based clustering algorithm for the CYGNO data analysis. JINST 2020, 15, T12003. [CrossRef]

32. Ester, M.; Kriegel, H.P.; Sander, J.; Xu, X. A Density-based Algorithm for Discovering Clusters a Density-based Algorithm for Discovering Clusters in Large Spatial Databases with Noise. In Proceedings of the Second International Conference on Knowledge Discovery and Data Mining, Oregon, Portland, 2-4 August 1996; pp. 226-231.

33. Baracchini, E.; Benussi, L.; Bianco, S.; Capoccia, C.; Caponero, M.A.; Cavoto, G.; Cortez, A.; Costa, I.A.; Marco, E.D.; D’Imperio, G.; et al. Identification of low energy nuclear recoils in a gas TPC with optical readout. arXiv 2020, arXiv:2007.12508.

34. Abbaneo, D.; Abbas, M.; Abbrescia, M.; Abdalla, H.; Ahmad, A.; Ahmed, A.; Ahmed, W.; Ali, C.; Asghar, I.; Aspell, P.; et al. Layout and Assembly Technique of the GEM Chambers for the Upgrade of the CMS First Muon Endcap Station. Nucl. Instrum. Meth. A 2019, 918, 67-75. [CrossRef]

35. Battat, J.B.R.; Daw, E.; Dorofeev, A.; Ezeribe, A.C.; Fox, J.R.; Gauvreau, J.-L.; Gold, M.; Harmon, L.; Harton, J.; Lafler, R.; et al. Reducing DRIFT Backgrounds with a Submicron Aluminized-Mylar Cathode. Nucl. Instrum. Meth. A 2015, 794, 33-46. [CrossRef]

36. Agostinelli, S.; Allison, J.; Amako, K.; Apostolakis, J.; Araujo, H.; Arce, P.; Asai, M.; Axen, D.; Banerjee, S.; Barrand, G.; et al. GEANT4: A Simulation toolkit. Nucl. Instrum. Meth. A 2003, 506, 250-303. [CrossRef]

37. Baracchini, E.; Benussi, L.; Bianco, S.; Capoccia, C.; Caponero, M.; Cavoto, G.; Cortez, A.; Costa, I.A.; Marco, E.D.; D’Imperio, G.; et al. CYGNO: A gaseous TPC with optical readout for dark matter directional search. JINST 2020, 15, C07036. [CrossRef]

38. Gonzalez-Diaz, D.; Monrabal, F.; Murphy, S. Gaseous and dual-phase time projection chambers for imaging rare processes. Nucl. Instrum. Meth. A 2018, 878, 200-255. [CrossRef]

39. Costa, E.; Soffitta, P.; Bellazzini, R.; Brez, A.; Lumb, N.; Spandre, G. An efficient photoelectric x-ray polarimeter for the study of black holes and neutron stars. Nature 2001, 411, 662-665. [CrossRef]

40. Beceiro-Novo, S.; Ahn, T.; Bazin, D.; Mittig, W. Active targets for the study of nuclei far from stability. Prog. Part. Nucl. Phys. 2015, 84, 124-165. [CrossRef]

41. Pomorski, M.; Pfützner, M.; Dominik, W.; Grzywacz, R.; Stolz, A.; Baumann, T.; Berryman, J.S.; Czyrkowski, H.; DÄ browski, R.; Fijałkowska, A.; et al. Proton spectroscopy of Ni48,Fe46, and Cr44. Phys. Rev. C 2014, 90, 014311. [CrossRef] 